\title{
Ozone exposure is associated with acute changes in inflammation, fibrinolysis, and endothelial cell function in coronary artery disease patients
}

\author{
Jaime E. Mirowsky ${ }^{1,2^{*}}$, Martha Sue Carraway ${ }^{3}$, Radhika Dhingra ${ }^{4}$, Haiyan Tong ${ }^{4}$, Lucas Neas ${ }^{4}$, David Diaz-Sanchez ${ }^{4}$, \\ Wayne Cascio ${ }^{4}$, Martin Case ${ }^{4}$, James Crooks ${ }^{5,6,7}$, Elizabeth R. Hauser ${ }^{8,9,10}$, Z. Elaine Dowdy ${ }^{8}$, William E. Kraus ${ }^{8,11}$ \\ and Robert B. Devlin ${ }^{4}$
}

\begin{abstract}
Background: Air pollution is a major risk factor for cardiovascular disease, of which ozone is a major contributor. Several studies have found associations between ozone and cardiovascular morbidity, but the results have been inconclusive. We investigated associations between ozone and changes across biological pathways associated with cardiovascular disease.

Methods: Using a panel study design, 13 participants with coronary artery disease were assessed for markers of systemic inflammation, heart rate variability and repolarization, lipids, blood pressure, and endothelial function. Daily measurements of ozone and particulate matter $\left(\mathrm{PM}_{2.5}\right)$ were obtained from central monitoring stations. Single (ozone) and two-pollutant (ozone and $\mathrm{PM}_{2.5}$ ) models were used to assess percent changes in measurements per interquartile ranges of pollutants.

Results: Per interquartile increase in ozone, changes in tissue plasminogen factor $(6.6 \%, 95 \%$ confidence intervals $(\mathrm{Cl})=0$. 4, 13.2), plasminogen activator inhibitor-1 $(40.5 \%, 95 \% \mathrm{Cl}=8.7,81.6)$, neutrophils $(8.7 \% 95 \% \mathrm{Cl}=1.5,16.4)$, monocytes (10. $2 \%, 95 \% \mathrm{Cl}=1.0,20.1)$, interleukin-6 $(15.9 \%, 95 \% \mathrm{Cl}=3.6,29.6)$, large-artery elasticity index $(-19.5 \%, 95 \% \mathrm{Cl}=-34.0,-1.7)$, and the baseline diameter of the brachial artery $(-2.5 \%, 95 \% \mathrm{Cl}=-5.0,0.1)$ were observed. These associations were robust in the two-pollutant model.

Conclusions: We observed alterations across several pathways associated with cardiovascular disease in 13 coronary artery disease patients following ozone exposures, independent of $\mathrm{PM}_{2.5}$. The results support the biological plausibility of ozone-induced cardiovascular effects. The effects were found at concentrations below the EPA National Ambient Air Quality Standards for both ozone and $\mathrm{PM}_{2.5}$.
\end{abstract}

Keywords: Ozone, Air pollution, Cardiovascular, Inflammation, Coronary artery disease, Panel study

\section{Background}

Air pollution is a major and independent environmental risk factor for cardiovascular disease [1]. Epidemiological studies suggest that the strongest associations are between particulate matter (PM) and cardiovascular morbidity and mortality $[2,3]$; however, recent work suggests that ozone also may be associated with negative

\footnotetext{
* Correspondence: jmirowsk@esf.edu

'Department of Chemistry, SUNY College of Environmental Science and Forestry, 1 Forestry Drive, Syracuse, NY 13210, USA

${ }^{2}$ Curriculum in Toxicology, University of North Carolina, Chapel Hill, NC, USA Full list of author information is available at the end of the article
}

cardiovascular health effects including coronary death, cardiac arrest, and ischemic stroke [4-6]. To clarify some contrary findings $[7,8]$ and establish biological plausibility, work is needed to establish potential mechanisms mediating ozone's adverse cardiovascular health effects [9].

Clinical and toxicological studies have established several possible mechanisms by which PM adversely impacts the cardiovascular system, thus providing biologic plausibility for the epidemiologic studies; similar approaches have begun to be used with ozone using controlled human exposure studies. Under controlled conditions, increases 
in systemic pro-inflammatory markers were observed in 26 healthy human participants following exposures to ozone and clean air $[10,11]$. In addition, researchers have found changes in fibrinolysis markers such as plasminogen activator inhibitor-1, plasminogen, and D-dimer, when young healthy participants were exposed to ozone $[11,12]$. Further, researchers have begun looking at changes in cardiovascular biomarkers in larger epidemiological work to support associations between ozone exposure and cardiovascular morbidity and mortality. In 1536 people living in Stockholm, changes in fibrinogen were associated with short-term ambient ozone exposures [13]. Blood pressure, platelet activation markers, and arterial stiffness were also found to be altered with ozone exposures in healthy adults living in China [14]. Additional research of men participating in the Normative Aging Study found changes in heart rate variability parameters with exposures to ambient ozone levels [15].

Some populations may experience enhanced adverse air pollutant-associated health effects: children, the elderly, the obese, and those with underlying disease. In studies of subjects with a concurrent history of cardiovascular disease, air pollution exposure has been associated with greater inflammation, coagulation, and decreases in heart rate variability $[16,17]$. In our previously conducted work in a cohort of coronary artery disease (CAD) patients, ambient ozone exposure was associated with changes in plasma metabolite levels [18]. This work suggests that metabolic processes may contribute to or mediate cardiovascular outcomes due to air pollutant exposure.

In order to expand upon this work we undertook a detailed panel study to assess whether there are alterations in several critical cardiovascular disease-associated biological pathways associated with acute ozone exposures. We recruited 13 volunteers with CAD to assess whether changes in endothelial function, fibrinolysis, inflammation, lipids, heart rate variability, and repolarization are observed following acute ozone exposure. In the interest of studying the effects of ozone in the context of particulate matter with a diameter of less than $2.5 \mu \mathrm{m}\left(\mathrm{PM}_{2.5}\right)$ and to address potential confounding, we used a two-pollutant model to control for PM concentrations collected from central monitoring stations [19]. The results of this work may provide biological plausibility in support of the concept that ozone induces adverse cardiovascular effects in susceptible populations.

\section{Methods}

\section{Study population and design}

The source population for this study was the Duke University Medical Center CATHeterization GENetics (CATHGEN) cohort of nearly 10,000 individuals [20]. Duke University is located in central North Carolina in the city of Durham. To participate in CATHGEN, patients were between 40 and 75 years of age and had undergone a cardiac catheterization between 2001 and 2010 at a Duke University Hospital.

Starting with residents that resided within a reasonable commuting distance to the U.S. Environmental Protection Agency's Human Studies Facility in Chapel Hill, NC, 448 letters were mailed out to participants enrolled in CATHGEN. The only exclusion criterion for not receiving a letter of invitation to participate was unstable angina and congestive heart failure. Fifty-four participants responded to the mailed letters. Of those, 15 participants meeting the severity criteria were enrolled for the current study. These volunteers were required to have a stable clinical status, documented coronary artery disease (> 75 occlusion in one major coronary vessel), a stable medication regimen over 3 months prior to enrollment, and an electrocardiogram demonstrating normal sinus rhythm. Exclusion criteria for the current study also included hematocrit $<34 \%$, current smoking or smoking history within 1 year of study (defined as more than one pack of cigarettes in the past year), sustained cardiac arrhythmias, presence of a pacemaker or implanted cardioverter-defibrillator, systolic blood pressure $(\mathrm{SBP}) \geq 150 \mathrm{mmHg}$ or $\leq 90 \mathrm{mmHg}$ or diastolic blood pressure $(\mathrm{DBP}) \geq 100 \mathrm{mmHg}$, known vascular obstruction of the upper extremities, unstable angina, moderate to severe chronic pulmonary disease (as determined by spirometry demonstrating $<60 \%$ predicted value for forced vital capacity (FVC) or forced expiratory volume in $1 \mathrm{~s}\left(\mathrm{FEV}_{1}\right)$ and including chronic obstructive pulmonary disease, pulmonary fibrosis, moderate to severe asthma, aortic stenosis), recent (past 6 months) myocardial infarction, cerebrovascular accident (i.e. stroke) or admission for heart failure, recent (past 6 months) vascular intervention/bypass surgery, or current pregnancy. Participants were also asked to refrain from vigorous exercise on study mornings and would be temporarily excluded from participation if they experienced a respiratory tract infection within the preceding 4 weeks or had a recent or recurring exposure to pollutants or irritants. All medications were evaluated by the study physician.

Each participant visited the U.S. Environmental Protection Agency's Human Studies Facility for two consecutive days for up to 10 weeks, between May 2012 and April 2014. The Human Studies Facility is approximately 8 miles southwest of Duke University. Upon arrival on the first study day, the subject's medical history was reviewed, vital signs were assessed, and the subject was outfitted with a Holter monitor which they would wear for the next $24 \mathrm{~h}$. The following day, under fasting conditions, the subject was assessed for biomarkers present in blood, heart rate variability and repolarization measured by Holter monitoring, blood pressure, and endothelial function measured 
by brachial artery ultrasound and pulse wave analysis. To minimize day-of-week effects, each subject was always studied on the same 2 days of the week. Written informed consent was given by all participants prior to enrollment, and the study was approved by the Duke University Institutional Review Board, the University of North Carolina at Chapel Hill Institutional Review Board, and the U.S. Environmental Protection Agency (EPA) Human Protocols Office.

\section{Clinical measurements}

\section{Peripheral venous blood samples}

Approximately $50 \mathrm{~mL}$ of venous blood was obtained from each subject. A portion of fresh blood was sent to a clinical laboratory (Lab Corp., Burlington, NC, USA) for analysis of differential blood cell counts, as well as quantification of blood lipids. The remaining blood samples were stored at $-80{ }^{\circ} \mathrm{C}$ prior to analysis.

Commercially available multiplex kits (Meso Scale Diagnostics, Gaithersburg, MD) were used to quantify levels of C-reactive protein (CRP), serum amyloid A (SAA), soluble intercellular adhesion molecule (sICAM), soluble vascular adhesion molecule (sVCAM), interleukin 1-beta (IL-1 $\beta$ ), interleukin-6 (IL-6), interleukin-8 (IL-8), and tumor necrosis factor-alpha (TNF- $\alpha$ ) (SECTOR ${ }^{\circ}$ Imager 2400, Meso Scale Diagnostics). All other assays (D-dimer, tissue plasminogen factor (tPA), von Willebrand factor (vWF), plasminogen activator inhibitor-1 (PAI-1), and plasminogen) were measured using MesoScale multiarray plates as per manufacturers' instructions.

\section{Brachial artery ultrasound}

Based on the guidelines by Corretti et al. [21], brachial artery ultrasound (BAU) was measured to evaluate endothelial cell function using a $15 \mathrm{MHz}$ transducer interfaced with an Acuson Sequoia 512 ultrasound machine (Siemens Healthcare, Malvern, PA, USA). As described earlier [22], resting blood pressure and the diameter of the brachial artery were measured at baseline, and the baseline diameter of the brachial artery (BAD) was also measured during reactive hyperemia for quantification of flow-mediated dilatation (FMD).

With the volunteer laying supine, a pneumatic tourniquet was placed around the right arm distal to the brachial artery. R-wave gated baseline images of the artery were acquired after $15 \mathrm{~min}$. The cuff was then inflated to a pressure of $50 \mathrm{mmHg}$ above the participant's SBP for $5 \mathrm{~min}$. The cuff was abruptly deflated to cause a hyperemia reaction. Images of the brachial artery were acquired for $90 \mathrm{~s}$, stored in a digital format, and subsequently analyzed. Arterial diameter from the lumenintimal interfaces of the proximal and distal walls was measured using customized software (Brachial Tools, Medical Imaging Applications, LLC, Coralville, IA,
USA). Data from at least three consecutive end-diastolic frames were averaged for each baseline measurement and from at least three frames at maximal dilatation during reactive hyperemia. Changes in diameter caused by reactive hyperemia (endothelium-dependent vasodilatation) were expressed as a percent change in vessel diameter from their respective baselines.

\section{Pulse wave analysis}

Arterial elasticity was measured by the contour analysis of the arterial pressure waveform (pulse wave) using the HDI/PulseWave CR-2000 Research Cardiovascular Profiling system (Hypertension Diagnostics Inc., Eagan, MN, USA) as previously described [22]. Three assessments of arterial compliance were obtained and averaged. Measurements included the large-artery elasticity index (LAEI) and small-artery elasticity index (SAEI). The system gathered and analyzed a 30-s analog tracing of the radial artery waveforms digitized at 200 samples/s. A beat determination was made during the 30-s time period to determine systole, peak systole, onset of diastole, and end of diastole. Representative averaged waveforms of individual beats were analyzed using a parameterestimating algorithm [23] to fit a multiplexed model [24]. Endothelial dysfunction is associated with decreasing elasticity indices.

\section{Holter monitoring}

Continuous ambulatory electrocardiograms (ECGs) were placed on each subject during the first day of each week's two-day visit and were collected for $24 \mathrm{~h}$ periods using a Mortara H12+ 12-Lead ECG Recorder (Mortara Instrument Co., Milwaukee, WI) sampling at $180 \mathrm{~Hz}$. During the second day, the subjects were asked to recline in a dark place, and data was collected for $30 \mathrm{~min}$. A trained nurse manually inspected and edited the sequence of ECG complexes to ensure proper labeling. RR-intervals that were more or less than $20 \%$ of the previous RR-interval were defined as abnormally long or short intervals and were interpolated using Mortara algorithms. Subsequent heart rate variability (HRV) indices for both the time and frequency domains were calculated.

Time-domain measurements were calculated over the full $24 \mathrm{~h}$ span, while a $5 \mathrm{~min}$ segment during the end of the resting period was used for the calculation of the frequency-domain and repolarization indices. Timedomain measurements included standard deviation of the normal-to-normal (SDNN) and root-mean squared of successive differences (rMSSD). Frequency-domain measurements included low frequency (LF, 0.04$0.15 \mathrm{~Hz}$ ), high frequency (HF, 0.15-0.40 Hz), low-tohigh frequency power (LF:HF), and the sum of the power spectrum density (PSD). 
Markers of cardiac repolarization were assessed by measuring the QT interval, which is it the measurement from the beginning of the QRS complex to the end of the T wave; we corrected the QT interval for heart rate (QTc). T wave complexity was measured in each beat by principal component analysis based on all 12 leads and averaged. QRS complexity and P wave complexity were calculated with Mortara software.

\section{Air pollution and meteorological measurements}

Daily $24 \mathrm{~h}$ measurements of ozone and $\mathrm{PM}_{2.5}$ were calculated from hourly pollutant data averaged between $9 \mathrm{AM}$ to $8 \mathrm{AM}$; this data was obtained from a central air monitoring station (Millbrook) located approximately $44 \mathrm{~km}$ (27 miles) from the EPA Human Studies Facility and operated by the State of North Carolina. Two visits used pollutant data from a different central monitor (Durham Armory) due to missing values; this location was approximately $18 \mathrm{~km}$ (11 mi) from the EPA Human Studies Facility. Concentrations were obtained for each clinic day, as well as for 4 days prior. Daily temperature, relative humidity, and pressure were also obtained from the Millbrook central monitoring station.

\section{Statistical analysis}

The study was conducted as a panel study with four to ten repeated measurements per participant. Thus, every subject acted as his/her own control, limiting the need for an adjustment for subject characteristics. Data were analyzed using the $\mathrm{R}$ statistical package (Version 3.3.0) using both a single (ozone) and two-pollutant (ozone and $\mathrm{PM}_{2.5}$ ) model. For the analyses, we used additive mixed models with a random subject effect that diminished the need for an adjustment for subject characteristics. Daily temperature and relative humidity corresponding to the lag of the pollutant were selected as covariates a priori, and we adjusted for seasonal trends using a natural spline. To determine the additional benefit of including a five-day average of barometric pressure as a covariate, models including and excluding the pressure term were compared on the basis of Akaike Information Criterion (AIC) and changes to the ozone coefficient. For this work, the ozone exposure was considered either as an immediate (lag 0), delayed (lag 1 to 4 ), or cumulative (5 day moving average, 5dMA) linear effect, similar to our previously published work [25]. All outcomes were log transformed prior to analysis, are reported as percent change from the mean of the measured outcome per unit interquartile range (IQR) of exposure, and statistical significance was set at $p<0.05$ for the percent change from the mean of the measured outcome per unit IQR of exposure.

\section{Results}

Of the 15 participants that were recruited for this study, two completed less than three study sessions and were not included in the final analysis (Table 1). The subjects' ages ranged from 53 to 68 years. Although inclusion in the study was open to both men and women, only men responded to advertisements and successfully passed the screening criteria. Most of the participants were taking medication: six subjects reported taking medication for diabetes, nine subjects were taking beta blockers, and twelve were taking HMG CoA reductase inhibitors (statins). Of the 13 subjects, five had experienced a previous myocardial infarction and nine had hypertension. Although subjects were excluded for being current smokers or smoking more than one pack of cigarettes 1 year before enrolling in this study, seven participants had a past history of smoking. Nine participants completed ten visits, two participants completed nine visits, one participant completed five visits, and one participant completed four visits. Altogether there were 117 exposure days analyzed (Fig. 1a).

Air pollution and meteorological measurements are shown in Table 2. Ozone concentrations during the entire study period (May 30, 2012-April 29, 2014) ranged from 0.002 to $0.063 \mathrm{ppm}$, with an interquartile range of $0.014 \mathrm{ppm}$ and a mean value of $0.026 \pm 0.010 \mathrm{ppm}$. Ozone concentrations at the Millbrook and Armory central monitoring stations were highly correlated

Table 1 Participant characteristics $(n=13)$

\begin{tabular}{ll}
\hline Characteristics $(\mathrm{n}=13)$ & Value \\
\hline Age (years) & $63(53-68)$ \\
Males & $13(100 \%)$ \\
Race & $10(77 \%)$ \\
Caucasian & $3(23 \%)$ \\
Black & \\
Current health status & $31(26-38)$ \\
BMl (kg/m $\left.{ }^{2}\right)$ & $130(102-141)$ \\
Systolic blood pressure & $77(65-90)$ \\
Diastolic blood pressure & $1(8 \%)$ \\
Asthma & \\
Current medication use & $6(46 \%)$ \\
Diabetes medication & $9(69 \%)$ \\
Beta blockers & $12(92 \%)$ \\
Statins & \\
Past health status & $5(38 \%)$ \\
Previous Ml & $9(69 \%)$ \\
Previous hypertension & $7(54 \%)$ \\
Past smokers &
\end{tabular}

Values expressed as either mean (range) or number (\%). BMI body mass index, MI myocardial infarction 
(Spearman correlation coefficient $=0.92 ; p<0.0001)$. $\mathrm{PM}_{2.5}$ mass concentrations during the study period ranged from 1.0 to $28.2 \mu \mathrm{g} / \mathrm{m}^{3}$, with an interquartile range of $5.4 \mu \mathrm{g} / \mathrm{m}^{3}$ and a mean value of $10.9 \pm 4.5 \mu \mathrm{g} /$ $\mathrm{m}^{3}$. Ozone and $\mathrm{PM}_{2.5}$ concentrations were not significantly correlated (Spearman correlation coefficient $=0.05$; $p=0.19)$. Temperature and humidity fluctuations during the study period ranged from 18.4 to $91.1^{\circ} \mathrm{F}$ and 26.5 to $96.3 \%$, respectively. In this paper, we describe the association of ozone with various cardiovascular outcomes, using both a one- (ozone) and two-pollutant (ozone and $\left.\mathrm{PM}_{2.5}\right)$ model. The results for all endpoints are shown in Additional file 1: (Table S1). Associations between $\mathrm{PM}_{2.5}$ and cardiovascular outcomes will be described elsewhere. Figure 1 shows the daily ozone concentrations during the nearly two-year duration of the study and at the date of each subject's clinical visits, which were spread out across various seasons to get a range of ozone concentrations for this work. The maximum observed ozone and $\mathrm{PM}_{2.5}$ concentrations during the study duration were below the EPA National Ambient Air Quality Standards, which are $0.070 \mathrm{ppm}$ for ozone $(8 \mathrm{~h})$ and $35 \mu \mathrm{g} / \mathrm{m}^{3}$ for $\mathrm{PM}_{2.5}(24 \mathrm{~h})$.

Large artery elasticity index (LAEI) decreased with increasing ozone concentrations (Fig. 2). There was a significant $19.5 \%$ decrease for the 5 day moving average (95\% confidence intervals $(\mathrm{CI})=-34.0,-1.7 ; p=0.03)$, and a borderline significant $11.7 \%$ decrease with a lag of 4 days $(95 \% \mathrm{CI}=-22.1,0.0 ; p=0.05)$. LAEI is a measure of arterial compliance, defined as the ability of an artery to expand and recoil with cardiac pulsation and relaxation. A decrease in compliance results in a stiffening of the artery and is a risk factor for atherosclerosis. For SAEI, there was a trend for an increase with lags of 2 and 3 days. There was a $2.5 \%$ decrease in the baseline diameter of the brachial artery (BAD) associated with ozone with a 2 day lag $(95 \% \mathrm{CI}=-5.0,0.1 ; p=0.06)$. This was counterbalanced with a significant $3.5 \%$ increase with a 4 day lag $(95 \% \mathrm{CI}=1.2,5.9 ; p<0.01$; Fig. 3). No significant changes were observed for FMD (Additional file 1: Table S1). There was also a 3.3\% marginally significant decrease in diastolic blood pressure with a 2 day lag $(95 \% \mathrm{CI}=-6.6,0.2 ; p=0.07)$. Similar decreases in blood pressure associated with ozone exposures have been reported by others [26].

Factors attributed to clotting and fibrinolysis included tissue plasminogen factor (tPA), plasminogen activator inhibitor-1 (PAI-1), von Willebrand factor (vWF), plasminogen, and D-dimer. Ozone was associated with a $6.6 \%$ increase in tPA with a 3 day lag $(95 \% \mathrm{CI}=0.4$, 13.2; $p=0.04$ ), and a near-significant $6.3 \%$ increase with a 4 day lag $(95 \% \mathrm{CI}=-0.1,13.1 ; p=0.05$; Fig. 4$)$, per IQR of ozone. A $20.0 \%$ increase in PAI- 1 with a 2 day lag $(95 \% \mathrm{CI}=0.8,42.8 ; p=0.04)$, and a $40.5 \%$ increase with a 5 day moving average were also observed (95\% $\mathrm{CI}=8.7,81.6 ; p=0.01)$; there were near-significant increases with $3(95 \% \mathrm{CI}=-2.3,35.0 ; p=0.09)$ and 4 (95\% CI $=-2.1,35.6 ; p=0.09)$ day lags. PAI-1 and tPA are associated the fibrinolysis pathway. No significant changes were observed for von Willebrand factor, plasminogen, and D-dimer (Additional file 1: Table S1).
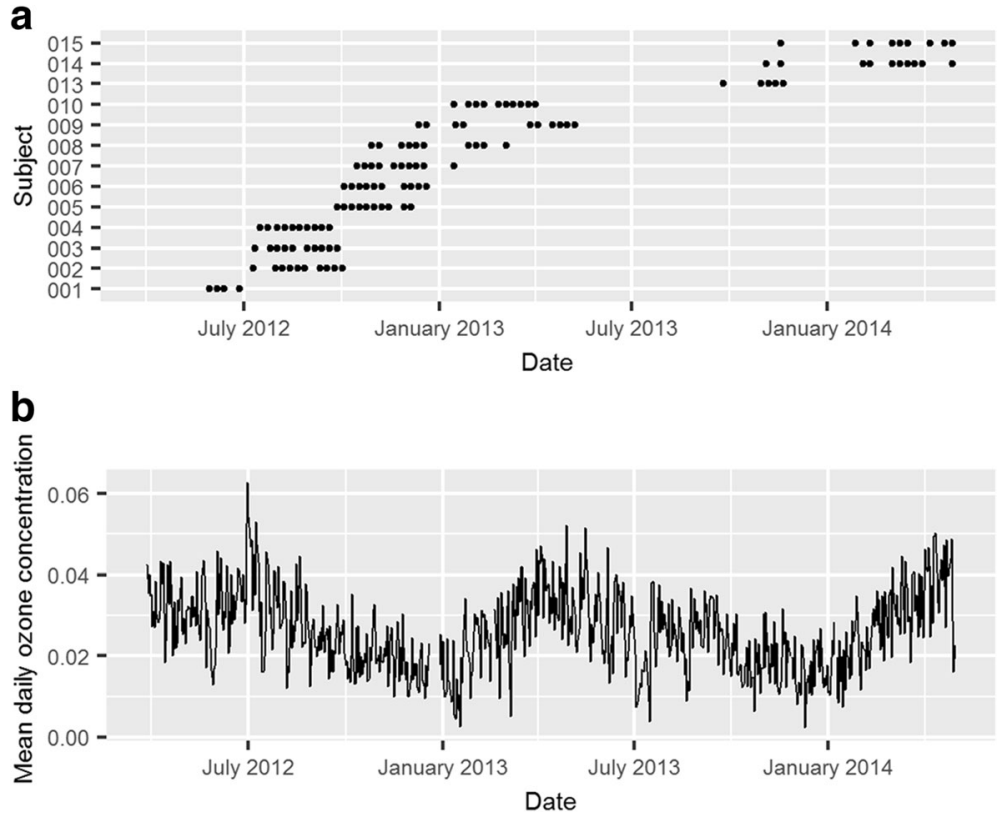

Fig. 1 Patient visits and mean daily ozone concentrations (ppm) between May 30, 2012-April 29, 2014. a Data points represent the days the patients visited the Human Studies Facility. b Daily 24-h ozone (ppm) concentrations (9 AM to 8 AM) calculated from the Millbrook central monitor 
Table 2 Average daily ozone concentrations during study period (May 30, 2012-April 29, 2014)

\begin{tabular}{llllllll}
\hline & Mean $\pm \mathrm{SD}$ & Minimum & 25th percentile & Median & 75th percentile & Maximum & IQR \\
\hline Ozone $(\mathrm{ppm})$ & $0.026 \pm 0.010$ & 0.002 & 0.019 & 0.025 & 0.033 & 0.063 & 0.014 \\
$\mathrm{PM}_{2.5}\left(\mathrm{\mu g} / \mathrm{m}^{3}\right)$ & $10.9 \pm 4.5$ & 1.0 & 7.8 & 9.9 & 13.2 & 28.2 & 5.4 \\
Temperature $\left({ }^{\circ} \mathrm{F}\right)$ & $59.1 \pm 16.0$ & 18.4 & 45.2 & 60.1 & 74.0 & 91.1 & 28.8 \\
Relative humidity $(\%)$ & $67.9 \pm 14.2$ & 26.5 & 57.9 & 70.0 & 78.4 & 96.3 \\
Barometric pressure $(\mathrm{hPa})$ & $1018.2 \pm 6.0$ & 1001.4 & 1014.0 & 1018.0 & 1021.9 & 1036.4 & 7.9 \\
\hline
\end{tabular}

$I Q R$ interquartile range, $P M$ particulate matter, $S D$ standard deviation

The number of neutrophils and monocytes, as well as the concentrations of IL- 6 , IL- 8, TNF- $\alpha$, CRP, SAA, sICAM, and sVCAM, were measured. Per IQR of ozone, an $8.7 \%$ increase in the number of neutrophils was observed with a 1 day lag $(95 \% \mathrm{CI}=1.5,16.4 ; p=0.02)$, an $8.4 \%$ increase was observed with a 2 day lag $(95 \%$ $\mathrm{CI}=1.0,16.3 ; p=0.03$ ), and a near-significant $11.2 \%$ increase was observed with the 5 day moving average (95\% CI $=-0.2,23.9 ; p=0.05$; Fig. 5). Ozone was also associated with a $10.2 \%$ increase in the number of monocytes following 1 day lag $(95 \% \mathrm{CI}=1.0,20.1$; $p=0.03)$. For IL-6, increases in $11.9 \%(95 \% \mathrm{CI}=-1.3$, $27.0 ; p=0.08)$ and $15.9 \%(95 \% \mathrm{CI}=3.6,29.6 ; p=0.01)$ per IQR of ozone were observed at 2 and 3 day lags, respectively; and a $5.9 \%$ increase in TNF- $\alpha$ was found following a 2 day lag $(95 \% \mathrm{CI}=-0.9,13.2 ; p=0.09$; Fig. 5). Finally, a $9.6 \%$ decrease in sVCAM was associated with increases 2 days prior to the collection of biological specimens $(95 \% \mathrm{CI}=-17.2,-1.2 ; p=0.03)$. No significant associations with ozone were observed for IL8, CRP, SAA, or sICAM (Additional file 1: Table S1).

As shown in Additional file 1: (Table S1), ozone was not significantly associated with changes in heart rate variability as measured by the standard deviation of the beat-to- beat interval (SDNN), root-mean squared of successive differences between adjacent NN intervals (rMSSD), low frequency domain (LF), high frequency domain (HF), and sum of the power spectrum density (PSD). Ozone was also not associated with measurements of repolarization including the duration of the QT interval (QTc), or complexity of the P-wave, T-wave, or QRS. We also found no associations between ozone and changes in lipids, including cholesterol, triglycerides, high density lipoprotein (HDL), or low density lipoprotein (LDL).

To determine if the associations observed with ozone were confounded by $\mathrm{PM}_{2.5}$, a two-pollutant model was used (Additional file 2: Table S2). For those outcomes that were associated with ozone in the single pollutant model, minimal (less than 10\%) changes were observed in the effect estimates when $\mathrm{PM}_{2.5}$ was included in the two-pollutant model, suggesting that the effects observed for ozone were specific to ozone and independent of $\mathrm{PM}_{2.5}$. In some cases, confidence intervals were slightly widened in the two-pollutant model, while in others they were slightly narrowed. Because of the limited sample size in this study, effect modification by age, gender, medication, and others could not be determined.

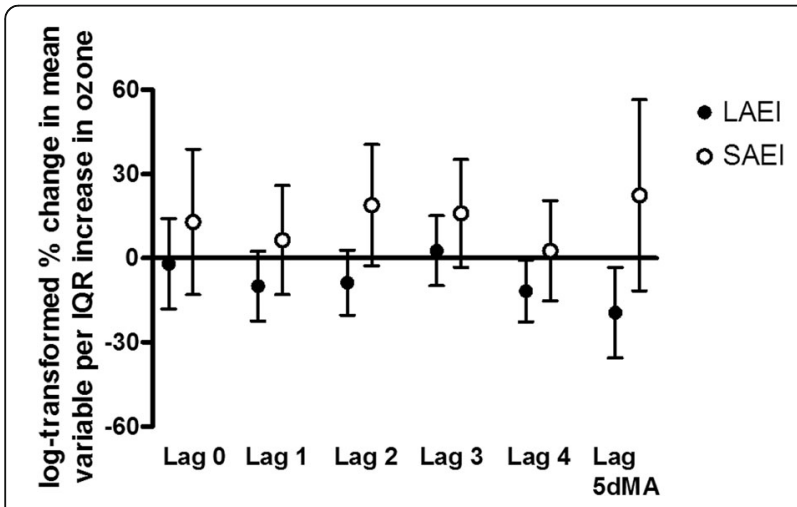

Fig. 2 Percent changes of elasticity indices with ambient ozone concentrations. Effect estimates $(95 \% \mathrm{Cl})$ were log-transformed, correspond to changes per IQR of ozone, and were adjusted for season, temperature, and humidity. $I Q R=$ interquartile range; $\mathrm{LAIE}=$ large artery elasticity index; SAEI = small artery elasticity index; $5 \mathrm{dMA}=5$ day moving average

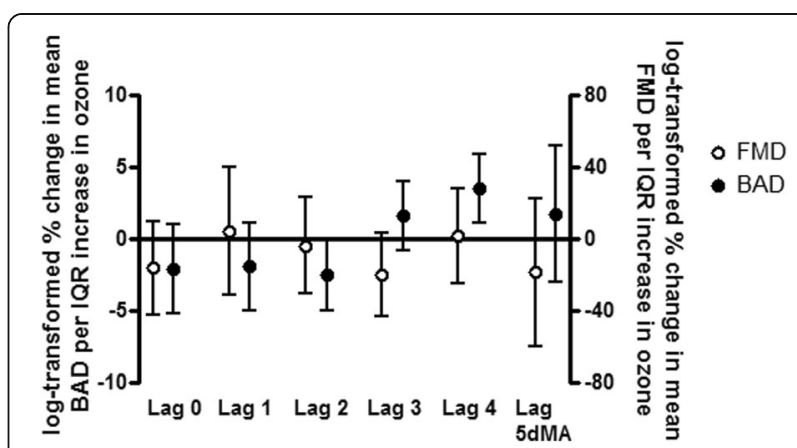

Fig. 3 Percent changes of endothelial function with ambient ozone concentrations. Effect estimates $(95 \% \mathrm{Cl})$ were log-transformed, correspond to changes per IQR of ozone, and were adjusted for season, temperature, and humidity. Effect estimates for FMD were also adjusted for the $5 \mathrm{dMA}$ barometric pressure. $\mathrm{IQR}=$ interquartile range; $F M D=$ flow-mediated dilatation; $B A D=$ baseline artery diameter; $5 \mathrm{dMA}=5$ day moving average 


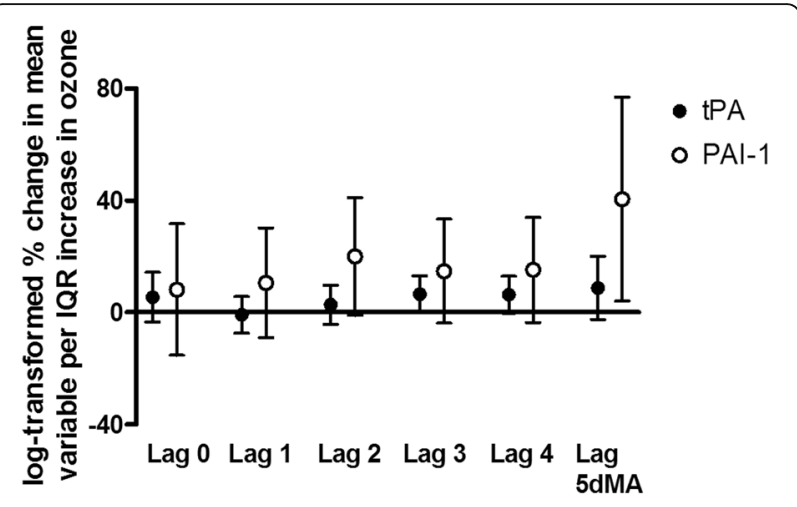

Fig. 4 Percent changes of clotting and fibrinolysis factors with ambient ozone concentrations. Effect estimates (95\% Cl) were log-transformed, correspond to changes per IQR of ozone, and were adjusted for season, temperature, and humidity. IQR = interquartile range; $\mathrm{tPA}=$ tissue plasminogen factor; PAI-1 = plasminogen activator inhibitor-1; $5 \mathrm{dMA}=5$ day moving average

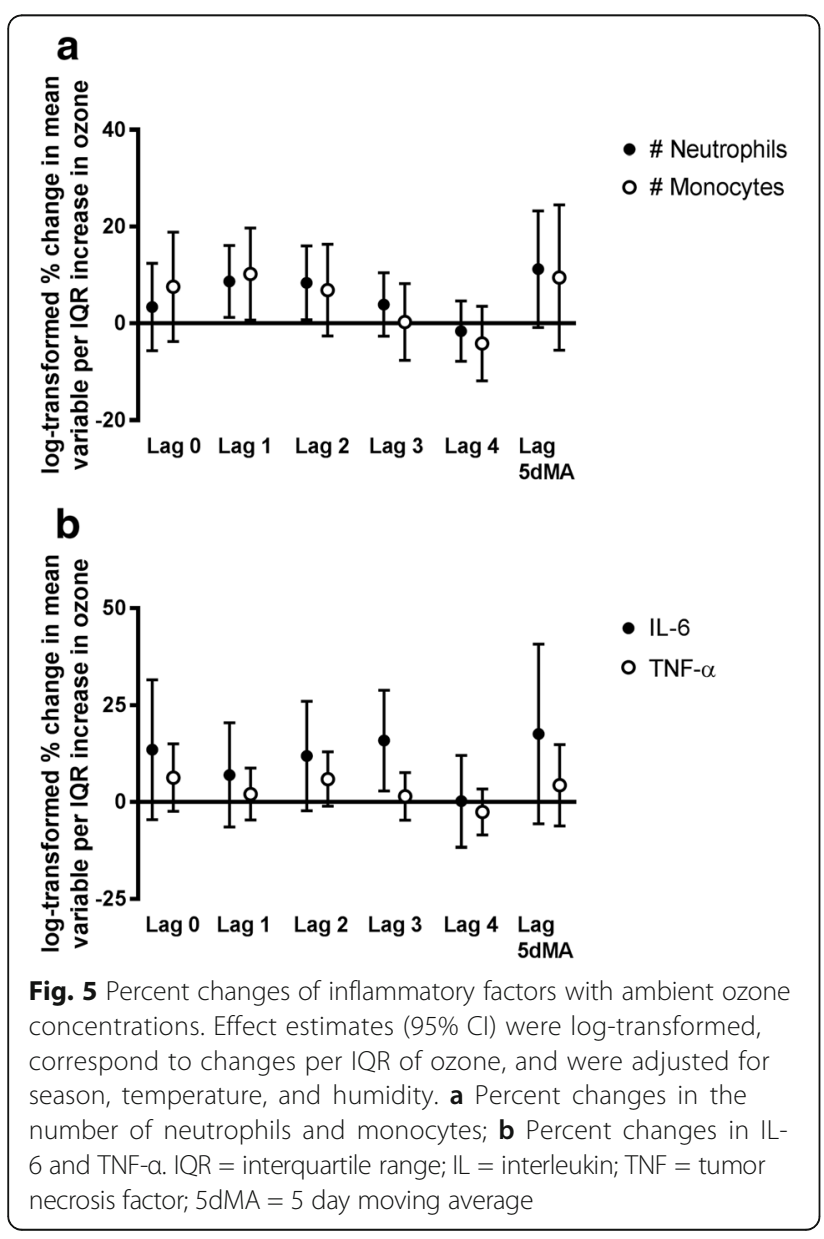

\section{Discussion}

In this panel study of a population with stable CAD, we observed associations between ambient ozone concentrations and changes in biological pathways involved in inflammation, fibrinolysis, and endothelial cell function. These changes were still evident when controlling for $\mathrm{PM}_{2.5}$ concentrations, suggesting that these results were independent of $\mathrm{PM}_{2.5}$ exposures.

Endothelium-derived mediators as well as vascular smooth muscle function regulate vascular tone structure, which can influence arterial stiffness and diameter. Arterial stiffness is the reduced ability of an artery to contract and expand during pressure changes [27]. One of the parameters used to describe arterial stiffness is arterial compliance, which is the measure of volume changes in a vessel in response to changes in arterial pressure [27]. Studies have found inverse associations between arterial compliance and age, glucose levels, smoking, hypertension, and metabolic syndrome [28, 29]. In the current study, we observed associations between ozone and a decrease in LAEI, indicating reduced arterial compliance. Past studies looking at other measures of arterial compliance have also reported negative associations with ozone exposure in elderly males as part of the VA Normative Aging Study Cohort [30] and in healthy adults [31]. In addition to LAEI, we observed an association between ozone and a decreased diameter of the brachial artery. Vasoconstriction caused by arterial narrowing can increase blood pressure, which could result in adverse outcomes in susceptible populations such as those with hypertension.

In this current work, we also found associations between ozone and increased levels of two critical components of the fibrinolysis pathway: tPA and PAI-1. The fibrinolytic pathway degrades blood clots that are formed during the normal course of living [32], and any process that inhibits this pathway increases the risk for adverse events associated with coagulation. When bound with tPA, plasminogen is converted to plasmin, which then degrades fibrin and dissolves clots. PAI-1 inhibits tPA, and increasing concentrations of PAI-1 are associated with decreases in fibrinolysis. Concentrations of PAI-1 in plasma are much higher than tPA, and assays quantifying tPA levels generally reflect the concentrations of tPA complexed with PAI-1 [33]. For this reason, increasing concentrations of tPA also can indicate reduced fibrinolysis [33]. Similar increases in tPA were observed in a previously conducted controlled human exposure study of ozone and clean air in healthy, young participants [11]. In a panel study of 76 young, healthy students in Taipei, increases in PAI-1 and tPA were found with 1- to 3- day averages of ambient ozone concentrations in both a single- and multi-pollutant model [34]. Taken as a whole, our data suggest that ozone can negatively impact the fibrinolysis pathway. 
A large body of research has shown associations between ozone and cellular and soluble makers of pulmonary inflammation $[35,36]$. In a previously conducted controlled ozone study, increases in the systemic inflammatory markers IL-1 $\beta$, IL-6, and TNF$\alpha$ were observed $24 \mathrm{~h}$ following ozone exposures compared to baseline values [12]. In a separate controlled study, plasma levels of IL-6 were elevated in both obese and non-obese non-smoking women after acute ozone exposures [37]. When 45 non-smoking adults enrolled in a panel study to assess the association of ambient air pollution and systemic inflammation, positive associations between IL- 6 and ozone were observed [38]. In this current work, we report an association between ozone and increased levels of cellular and soluble markers of vascular inflammation, as well as associations between ozone and increased numbers of monocytes, neutrophils, and two markers of inflammation: IL- 6 and TNF- $\alpha$. Clinically, increases in plasma IL- 6 and TNF- $\alpha$ have been associated with cardiovascular disease and disease outcomes [39].

Our findings in humans with CAD corroborate some experimental data from animals exposed to air pollution. When rats were exposed to $0.50 \mathrm{ppm}$ of ozone for $5 \mathrm{~h} /$ day for 2 days, increases in PAI-1 were observed [40]. However, in contrast, Farraj et al. [41] observed significant changes in heart rate variability parameters and blood lipids in spontaneously hypertensive rats exposed to various ozone concentrations; these changes were only observed after high ozone exposures $(0.8 \mathrm{ppm})$, and no changes in heart rate variability were found following lower exposures $(0.2 \mathrm{ppm})$. Therefore, it is possible that we did not observe alterations in heart rate variability due to the relatively low ozone concentration observed in the ambient environment compared with those used in laboratory studies.

We observed that several of the biological pathways disrupted by $\mathrm{PM}_{2.5}$ exposures may also be altered by ozone exposures. For example, we previously reported decreased levels of LAEI to be associated with $\mathrm{PM}_{2.5}$ in type 2 diabetics [22]. Additionally, increases in tPA [42] and systemic pro-inflammatory cytokines [43] are associated with particle exposures.

In contrast with studies looking at cardiovascular changes or blood lipids associated with $\mathrm{PM}_{2.5}$ exposures $[25,44]$, we did not observe associations between ozone and any marker of heart rate variability or repolarization, or in blood lipids. It is possible that medication use prevented us from seeing these effects. For example, 70\% of the participants were taking beta blocker medication which is known to interfere with HRV analysis [45], and a similar proportion were taking statin medications, which may impact air pollution-induced increases in blood lipids.
Lag effects may differ between ozone and $\mathrm{PM}_{2.5}$ as well. In a previous panel study of diabetic patients, we observed an association between $\mathrm{PM}_{2.5}$ and inflammatory changes at lags $2-3$, but endothelial function changes occurred with a 0 day lag $[22,25]$. In the current study, we did not observe rapid changes (i.e. those occurring with a 0 or 1 day lag) associated with ozone, with the exception being systemic inflammatory mediators. This is consistent with observations of rapid responses in human systemic inflammatory markers in laboratory-controlled conditions [11]. Similarly, Bind et al. [46] observed ozone-induced responses in pro-inflammatory mediators as early as 4 and $24 \mathrm{~h}$, which then decreased in intensity at later time points.

There are several strengths in this study. Unlike prior studies, we examined a significantly at-risk population those with active CAD. Further, given the recent interest in examining the effects of multiple pollutants simultaneously, we used a two-pollutant model to adjust for $\mathrm{PM}_{2.5}$ concentrations, observing ozone effects independent of $\mathrm{PM}_{2.5}$. We also studied the time-course of effects at several temporal lags relative to exposure. Last, via the repeated measures study design, we accounted for both between- and within-subject variability.

There were also several limitations. It is possible that our study may suffer from selection bias; inclusion in the CATHGEN cohort could be as a result of referral bias, enrollment biases, and those related to socioeconomic status and other unmeasured confounders. We also used central monitoring stations for our exposures, which may result in exposure misclassification. All of the subjects were taking at least one cardiovascular disease medication, which could have affected our ability to detect ozone-associated differences. Further, given the relatively small sample size, we were not able to assess for effect modification such as genotype, body mass index, and medication [22, 25].

\section{Conclusions}

In this panel study of ambient air pollution exposure in a population with extant coronary heart disease, we observed ozone-associated alterations in several pathways associated with cardiovascular morbidity and mortality: fibrinolysis, systemic inflammation, and vascular reactivity. The effects remained strong after controlling for $\mathrm{PM}_{2.5}$ concentrations. Future work will compare these effects with effects associated with $\mathrm{PM}_{2.5}$ in the same cohort. This study contributes new information regarding the mechanisms underlying the effects of ozone on cardiovascular risk. The observed effects were found at ambient concentrations below the EPA National Ambient Air Quality Standards for both ozone and $\mathrm{PM}_{2.5}$. 


\section{Additional files}

Additional file 1: Table S1. Percent changes of measured factors with ambient ozone concentrations. Effect estimates (95\% Cl) were logtransformed, correspond to changes per IQR of ozone, and were adjusted for season, temperature, and humidity. Effect estimates for SumPSD, LF:HF, LF, HF, FMD, and CRP were also adjusted for the $5 \mathrm{dMA}$ barometric pressure. $\mathrm{LAIE}=$ large artery elasticity index; $\mathrm{SAEI}=$ small artery elasticity index FMD $=$ flow-mediated dilatation; $B A D=$ baseline artery diameter $; \mathrm{SBP}=$ systolic blood pressure; $\mathrm{DBP}=$ diastolic blood pressure; $\mathrm{PPA}=$ tissue plasminogen factor; PAl-1 = plasminogen activator inhibitor-1; $\mathrm{VWF}=$ von Willebrand factor; $\mathrm{IL}=$ interleukin; $\mathrm{TNF}=$ tumor necrosis factor; $\mathrm{CRP}=\mathrm{C}$-reactive protein; SAA = serum amyloid A; sICAM = soluble intercellular adhesion molecule; sVCAM = soluble vascular adhesion molecule; $\mathrm{HDL}=$ high density lipoprotein; $\mathrm{LDL}=$ low density lipoprotein; $\mathrm{LF}=$ low frequency; HF = high frequency; PSD = power spectrum density; SDNN = standard deviation of the normalto-normal; rMSSD = root-mean squared of successive differences. ${ }^{*} p$ value < 0.10 for the percent change from the mean of the measured outcome per unit IQR of exposure, ${ }^{* *} p$ value $<0.05$ for the percent change from the mean of the measured outcome per unit IQR of exposure. (DOCX $21 \mathrm{~kb}$ )

Additional file 2: Table S2. Percent changes of measured factors with ambient ozone and $\mathrm{PM}_{2.5}$ concentrations using a two-pollutant model. Effect estimates $(95 \% \mathrm{Cl}$ ) were log-transformed, correspond to changes per IQR of the corresponding pollutants, and were adjusted for season, temperature, and humidity. Effect estimates for FMD were also adjusted for the 5dMA barometric pressure. $\mathrm{LAIE}=$ large artery elasticity index; SAEI = small artery elasticity index; $F M D=$ flow-mediated dilatation; $\mathrm{BAD}=$ baseline artery diameter; $\mathrm{IL}=$ interleukin; TNF = tumor necrosis factor; tPA = tissue plasminogen factor; PAl-1 = plasminogen activator inhibitor-1. ${ }^{*} p$ value $<0.10$ for the percent change from the mean of the measured outcome per unit IQR of exposure, ${ }^{* *} p$ value $<0.05$ for the percent change from the mean of the measured outcome per unit IQR of exposure. (DOCX $21 \mathrm{~kb}$ )

\section{Abbreviations}

5dMA: 5 day moving average; AIC: Akaike Information Criterion: BAD: Diameter of the brachial artery; BAU: Brachial artery ultrasound; CAD: Coronary artery disease; CATHGEN: CATHeterization GENetics; $\mathrm{Cl}$ : Confidence intervals; CRP: C-reactive protein; DBP: Diastolic blood pressure; ECG: Electrocardiograms; EPA: Environmental Protection Agency; $\mathrm{FEV}_{1}$ : Forced expiratory volume in 1s; FMD: Flow-mediated dilatation; FVC: Forced vital capacity; HDL: High density lipoprotein; HF: High frequency; HRV: Heart rate variability; IL-1 $\beta$ : Interleukin 1-beta; IL-6: Interleukin-6; IL8: Interleukin-8; IQR: Interquartile range; LAEI: Large-artery elasticity index; LDL: Low density lipoprotein; LF: Low frequency; PAI-1: Plasminogen activator inhibitor-1; PM: Particulate matter; PSD: Power spectrum density; QTc: QT interval corrected; rMSSD: Root-mean squared of successive differences; SAA: Serum amyloid A; SAEl: Small-artery elasticity index; SBP: Systolic blood pressure; SDNN: Standard deviation of the normal-tonormal; sICAM: Soluble intercellular adhesion molecule; sVCAM: Soluble vascular adhesion molecule; TNF-a: Tumor necrosis factor-alpha; tPA: Tissue plasminogen factor; vWF: Von Willebrand factor

\section{Acknowledgements}

All authors read and approved the final manuscript. The research described in this article has been reviewed by the National Health and Environmental Effects Research Laboratory, U.S. Environmental Protection Agency, and approved for publication. Approval does not signify that the contents necessarily reflect the views and policies of the Agency, nor does the mention of trade names of commercial products constitute endorsement or recommendation for use.

\section{Funding}

This work was supported by United States Environmental Protection Agency internal funds; the Environmental Protection Agency Cooperative Agreement with the Center for Environmental Medicine, Asthma, and Lung Biology at the University of North Carolina [CR83346301]; the National Institute of Environmental Health Sciences [T32ES007126]; University of North Carolina Golberg Fellowship; the National Institutes of Health [HL73042, HL36587, HL095987]; an award from the Neurosciences Education and Research Foundation (Encinitas, CA); and the Health Effects Institute [4946-RFPA10-3/14-7].

\section{Availability of data and materials}

The datasets used and/or analyzed during the current study are available from the corresponding author on reasonable request.

\section{Authors' contributions}

Conceptualization (DDS, MSC, RBD, WC, WEK); methodology (ERN, LN, MSC) formal analysis (JC, JEM, RD); investigation (HT, MSC, MC); resources $(E R H)$; data curation (ZED); writing (original draft preparation - JEM, RBD; review and editing - DDS, ERH, MSC, LN, WC, WEK); supervision (DDS, ERH, RBD, WEK); project administration (DDS, MSC, RBD); funding acquisition (DDS, WEK). All authors read and approved the final manuscript.

\section{Ethics approval and consent to participate}

Written informed consent was given by all participants prior to enrollment, and the study was approved by the Duke University Institutional Review Board, the University of North Carolina at Chapel Hill Institutional Review Board, and the U.S. Environmental Protection Agency (EPA) Human Protocols Office.

\section{Consent for publication}

Not applicable.

\section{Competing interests}

The authors declare that they have no competing interests.

\section{Publisher's Note}

Springer Nature remains neutral with regard to jurisdictional claims in published maps and institutional affiliations.

\section{Author details}

${ }^{1}$ Department of Chemistry, SUNY College of Environmental Science and Forestry, 1 Forestry Drive, Syracuse, NY 13210, USA. ${ }^{2}$ Curriculum in Toxicology, University of North Carolina, Chapel Hill, NC, USA. ${ }^{3}$ Department of Medicine, Pulmonary and Critical Care Medicine, Durham VA Medical Center, Durham, NC, USA. ${ }^{4}$ National Health and Environmental Effects Laboratory, US Environmental Protection Agency, Chapel Hill, NC, USA. ${ }^{5}$ Department of Biomedical Research, National Jewish Health, Denver, CO, USA. ${ }^{6}$ Division of Biostatistics and Bioinformatics, National Jewish Health, Denver, CO, USA. ${ }^{7}$ Department of Epidemiology, Colorado School of Public Health, Denver, CO, USA. ${ }^{8}$ Duke Molecular Physiology Institute, Duke University School of Medicine, Durham, NC, USA. ${ }^{9}$ Department of Biostatistics and Bioinformatics, Duke University School of Medicine, Durham, NC, USA.

${ }^{10}$ Cooperative Studies Program Epidemiology Center, Durham Veterans Affairs Medical Center, Durham, NC, USA. ${ }^{11}$ Division of Cardiology, Department of Medicine, School of Medicine, Duke University, Durham, NC, USA.

Received: 12 June 2017 Accepted: 23 October 2017

Published online: 21 November 2017

\section{References}

1. Brook RD, Rajagopalan S, Pope CA, Brook JR, Bhatnagar A, Diez-Roux AV, et al. Particulate matter air pollution and cardiovascular disease: an update to the scientific statement from the American Heart Association. Circulation. 2010;121(21):2331-78.

2. EPA. U: 2009 Final Report: Integrated Science Assessment for Particulate Matter. In. Edited by Agency USEP. Washington, DC; 2009.

3. Pope CA 3rd, Dockery DW. Health effects of fine particulate air pollution: lines that connect. J Air Waste Manage Assoc. 2006:56(6):709-42.

4. Nuvolone D, Balzi D, Pepe P, Chini M, Scala D, Giovannini F, et al. Ozone short-term exposure and acute coronary events: a multicities study in Tuscany (Italy). Environ Res. 2013;126:17-23.

5. Raza A, Bellander T, Bero-Bedada G, Dahlquist M, Hollenberg J, Jonsson M, et al. Short-term effects of air pollution on out-of-hospital cardiac arrest in Stockholm. Eur Heart J. 2014;35(13):861-8.

6. Suissa L, Fortier M, Lachaud S, Staccini P, Mahagne MH. Ozone air pollution and ischaemic stroke occurrence: a case-crossover study in nice, France. BMJ Open. 2013:3(12):e004060.

7. Goodman JE, Prueitt RL, Sax SN, Pizzurro DM, Lynch HN, Zu K, et al. Ozone exposure and systemic biomarkers: evaluation of evidence for adverse cardiovascular health impacts. Crit Rev Toxicol. 2015;45(5):412-52. 
8. Shah AS, Lee KK, McAllister DA, Hunter A, Nair H, Whiteley W, et al. Short term exposure to air pollution and stroke: systematic review and metaanalysis. BMJ. 2015:350, h1295.

9. Srebot V, Gianicolo EA, Rainaldi G, Trivella MG, Sicari R. Ozone and cardiovascular injury. Cardiovasc Ultrasound. 2009;7:30.

10. Arjomandi $\mathrm{M}$, Wong $\mathrm{H}$, Donde A, Frelinger J, Dalton S, Ching W, et al. Exposure to medium and high ambient levels of ozone causes adverse systemic inflammatory and cardiac autonomic effects. Am J Physiol Heart Circ Physiol. 2015;308(12):H1499-509.

11. Devlin RB, Duncan KE, Jardim M, Schmitt MT, Rappold AG, Diaz-Sanchez D. Controlled exposure of healthy young volunteers to ozone causes cardiovascular effects. Circulation. 2012;126(1):104-11.

12. Kahle JJ, Neas LM, Devlin RB, Case MW, Schmitt MT, Madden MC, et al. Interaction effects of temperature and ozone on lung function and markers of systemic inflammation, coagulation, and fibrinolysis: a crossover study of healthy young volunteers. Environ Health Perspect. 2015;123(4):310-6.

13. Panasevich $S$, Leander $K$, Rosenlund M, Ljungman $P$, Bellander $T$, de Faire $U$, et al. Associations of long- and short-term air pollution exposure with markers of inflammation and coagulation in a population sample. Occup Environ Med. 2009;66(11):747-53.

14. Day DB, Xiang J, Mo J, Li F, Chung M, Gong J, et al. Association of Ozone Exposure with Cardiorespiratory Pathophysiologic Mechanisms in healthy adults. JAMA Intern Med. 2017;

15. Park SK, O'Neill MS, Vokonas PS, Sparrow D, Schwartz J. Effects of air pollution on heart rate variability: the VA normative aging study. Environ Health Perspect. 2005;113(3):304-9.

16. Bartell SM, Longhurst J, Tjoa T, Sioutas C, Delfino RJ. Particulate air pollution, ambulatory heart rate variability, and cardiac arrhythmia in retirement community residents with coronary artery disease. Environ Health Perspect. 2013;121(10):1135-41.

17. Yue W, Schneider A, Stölzel M, Rückerl R, Cyrys J, Pan X, et al. Ambient source-specific particles are associated with prolonged repolarization and increased levels of inflammation in male coronary artery disease patients. Mutat Res. 2007;621(1-2):50-60

18. Breitner S, Schneider A, Devlin RB, Ward-Caviness CK, Diaz-Sanchez D, Neas LM, et al. Associations among plasma metabolite levels and short-term exposure to PM2.5 and ozone in a cardiac catheterization cohort. Environ Int. 2016;97:76-84.

19. Bell ML, McDermott A, Zeger SL, Samet JM, Dominici F. Ozone and short-term mortality in 95 US urban communities, 1987-2000. JAMA. 2004;292(19):2372-8.

20. Kraus WE, Granger CB, Sketch MH Jr, Donahue MP, Ginsburg GS, Hauser ER, et al. A guide for a cardiovascular genomics biorepository: the CATHGEN experience. J Cardiovasc Transl Res. 2015;

21. Corretti MC, Anderson TJ, Benjamin EJ, Celermajer D, Charbonneau F, Creager MA, et al. Guidelines for the ultrasound assessment of endothelialdependent flow-mediated vasodilation of the brachial artery: a report of the international brachial artery reactivity task force. J Am Coll Cardiol. 2002; 39(2):257-65.

22. Schneider A, Neas L, Herbst MC, Case M, Williams RW, Cascio W, et al. Endothelial dysfunction: associations with exposure to ambient fine particles in diabetic individuals. Environ Health Perspect. 2008;116(12):1666-74.

23. Finkelstein SM, Cohn JN. First- and third-order models for determining arterial compliance. J Hypertens Suppl. 1992;10(6):S11-4.

24. O'Rourke MF, Gallagher DE. Pulse wave analysis. Journal of hypertension supplement : official journal of the international society of. Hypertension. 1996;14(5):S147-57.

25. Schneider A, Neas LM, Graff DW, Herbst MC, Cascio WE, Schmitt MT, et al. Association of cardiac and vascular changes with ambient PM2.5 in diabetic individuals. Part Fibre Toxicol. 2010;7:14

26. Hoffmann B, Luttmann-Gibson H, Cohen A, Zanobetti A, de Souza C, Foley C, et al. Opposing effects of particle pollution, ozone, and ambient temperature on arterial blood pressure. Environ Health Perspect. 2012;120(2):241-6.

27. Cecelja M, Chowienczyk P. Role of arterial stiffness in cardiovascular disease. JRSM Cardiovasc Dis. 2012;1(4)

28. Li B, Gao H, Li X, Liu Y, Wang M. Correlation between brachial-ankle pulse wave velocity and arterial compliance and cardiovascular risk factors in elderly patients with arteriosclerosis. Hypertens Res. 2006; 29(5):309-14.

29. Ge JY, Li XL, Zhang HF, Xu Q, Tong M, Wang JG. Elasticity indices of large and small arteries in relation to the metabolic syndrome in Chinese. Am J Hypertens. 2008;21(2):143-7.
30. Mehta AJ, Zanobetti A, Koutrakis P, Mittleman MA, Sparrow D, Vokonas $P$, et al. Associations between short-term changes in air pollution and correlates of arterial stiffness: the veterans affairs normative aging study, 2007-2011. Am J Epidemiol. 2014;179(2):192-9.

31. CF W, Kuo IC, TC S, Li YR, Lin LY, Chan CC, et al. Effects of personal exposure to particulate matter and ozone on arterial stiffness and heart rate variability in healthy adults. Am J Epidemiol. 2010;171(12):1299-309.

32. Anglés-Cano E. Overview on fibrinolysis: plasminogen activation pathways on fibrin and cell surfaces. Chem Phys Lipids. 1994;67-68:353-62.

33. de Bono D. Significance of raised plasma concentrations of tissue-type plasminogen activator and plasminogen activator inhibitor in patients at risk from ischaemic heart disease. Br Heart J. 1994;71(6):504-7.

34. Chuang KJ, Chan CC, Su TC, Lee CT, Tang CS. The effect of urban air pollution on inflammation, oxidative stress, coagulation, and autonomic dysfunction in young adults. Am J Respir Crit Care Med. 2007:176(4):370-6.

35. EPA U. 2013 final report: integrated science assessment of ozone and related photochemical oxidants. Washington, DC: Edited by Agency USEP; 2013.

36. Nuvolone D, Petri D, Voller F. The effects of ozone on human health. Environ Sci Pollut Res Int. 2017.

37. Bennett WD, Ivins S, Alexis NE, Wu J, Bromberg PA, Brar SS, et al. Effect of obesity on acute ozone-induced changes in airway function, reactivity, and inflammation in adult females. PLoS One. 2016:11(8):e0160030.

38. Thompson AM, Zanobetti A, Silverman F, Schwartz J, Coull B, Urch B, et al. Baseline repeated measures from controlled human exposure studies: associations between ambient air pollution exposure and the systemic inflammatory biomarkers IL-6 and fibrinogen. Environ Health Perspect. 2010; 118(1):120-4.

39. Cesari M, Penninx BW, Newman AB, Kritchevsky SB, Nicklas BJ, Sutton-Tyrrell $K$, et al. Inflammatory markers and onset of cardiovascular events: results from the health ABC study. Circulation. 2003;108(19):2317-22.

40. Kodavanti UP, Thomas R, Ledbetter AD, Schladweiler MC, Shannahan JH, Wallenborn JG, et al. Vascular and cardiac impairments in rats inhaling ozone and diesel exhaust particles. Environ Health Perspect. 2011:119(3):312-8.

41. Farraj AK, Hazari MS, Winsett DW, Kulukulualani A, Carll AP, Haykal-Coates N, et al. Overt and latent cardiac effects of ozone inhalation in rats: evidence for autonomic modulation and increased myocardial vulnerability. Environ Health Perspect. 2012;120(3):348-54.

42. Tong H, Rappold AG, Caughey M, Hinderliter AL, Bassett M, Montilla T, et al. Dietary supplementation with olive oil or fish oil and vascular effects of concentrated ambient particulate matter exposure in human volunteers. Environ Health Perspect. 2015;123(11):1173-9.

43. Krishnan RM, Sullivan JH, Carlsten C, Wilkerson HW, Beyer RP, Bammler T, et al. A randomized cross-over study of inhalation of diesel exhaust, hematological indices, and endothelial markers in humans. Part Fibre Toxicol. 2013;10:7

44. Pieters $N$, Plusquin M, Cox B, Kicinski M, Vangronsveld J, Nawrot TS. An epidemiological appraisal of the association between heart rate variability and particulate air pollution: a meta-analysis. Heart. 2012;98(15):1127-35.

45. Niemelä MJ, Airaksinen KE, Huikuri HV. Effect of beta-blockade on heart rate variability in patients with coronary artery disease. J Am Coll Cardiol. 1994. 23(6):1370-7.

46. Bind MA, Baccarelli A, Zanobetti A, Tarantini L, Suh H, Vokonas P, et al. Air pollution and markers of coagulation, inflammation, and endothelial function: associations and epigene-environment interactions in an elderly cohort. Epidemiology. 2012;23(2):332-40.

\section{Submit your next manuscript to BioMed Central and we will help you at every step:}

- We accept pre-submission inquiries

- Our selector tool helps you to find the most relevant journal

- We provide round the clock customer support

- Convenient online submission

- Thorough peer review

- Inclusion in PubMed and all major indexing services

- Maximum visibility for your research

Submit your manuscript at www.biomedcentral.com/submit 